\title{
Structure of polyamidoamide dendrimers up to limiting generations: A mesoscale description
}

\author{
Prabal K. Maiti, ${ }^{1, a)}$ Youyong $\mathrm{Li}^{2}{ }^{2}$ Tahir Cagin, ${ }^{3, \mathrm{~b})}$ and William A. Goddard III ${ }^{2, \mathrm{c})}$ \\ ${ }^{1}$ Center for Condensed Matter Theory, Department of Physics, Indian Institute of Science, \\ Bangalore 560012, India \\ ${ }^{2}$ Materials and Process Simulation Center, California Institute of Technology, \\ Pasadena, California 91125, USA \\ ${ }^{3}$ Department of Chemical Engineering, Texas A\&M University, Texas 77843, USA
}

(Received 21 November 2008; accepted 23 February 2009; published online 8 April 2009)

\begin{abstract}
The polyamidoamide (PAMAM) class of dendrimers was one of the first dendrimers synthesized by Tomalia and co-workers at Dow. Since its discovery the PAMAMs have stimulated many discussions on the structure and dynamics of such hyperbranched polymers. Many questions remain open because the huge conformation disorder combined with very similar local symmetries have made it difficult to characterize experimentally at the atomistic level the structure and dynamics of PAMAM dendrimers. The higher generation dendrimers have also been difficult to characterize computationally because of the large size (294852 atoms for generation 11) and the huge number of conformations. To help provide a practical means of atomistic computational studies, we have developed an atomistically informed coarse-grained description for the PAMAM dendrimer. We find that a two-bead per monomer representation retains the accuracy of atomistic simulations for predicting size and conformational complexity, while reducing the degrees of freedom by tenfold. This mesoscale description has allowed us to study the structural properties of PAMAM dendrimer up to generation 11 for time scale of up to several nanoseconds. The gross properties such as the radius of gyration compare very well with those from full atomistic simulation and with available small angle $\mathrm{x}$-ray experiment and small angle neutron scattering data. The radial monomer density shows very similar behavior with those obtained from the fully atomistic simulation. Our approach to deriving the coarse-grain model is general and straightforward to apply to other classes of dendrimers. (C) 2009 American Institute of Physics. [DOI: 10.1063/1.3105338]
\end{abstract}

\section{INTRODUCTION}

Dendrimer polymers provide a unique and highly versatile set of properties because of the exquisite control of their multiplicity of internal and external binding sites. These properties have also led to considerable interest in application over fields ranging from medicine to environmental cleanup. ${ }^{1-5}$ Many studies, both experimental and theoretical, have been directed toward elucidating the size, distribution of surface sites, and character of the internal sites, the fluctuations in these quantities and how these properties depend on the presence of solvent and variation in solvent $p \mathrm{H}$. However many of these questions remain open because the huge conformation disorder combined with very similar local symmetries have made it difficult to characterize experimentally at the atomistic level the structure of the dendrimer. Similarly there are severe computational limitations using theory to answer such questions because of the large size (294 852 atoms for generation 11) and the huge number of conformations. The computational situation becomes formidable if we want to study its $p \mathrm{H}$ responsive behavior in explicit solvent to mimic the experimental scenarios.

\footnotetext{
${ }^{a)}$ Electronic mail: maiti@physics.iisc.ernet.in.

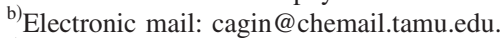

${ }^{c)}$ Electronic mail: wag@wag.caltech.edu.
}

Earlier we reported the structural properties of polyamidoamide (PAMAM) dendrimer up to generation 11 using a fully atomistic description. ${ }^{6}$ However the computational costs of these simulations (especially for higher generation) limited the time scales to a few hundreds picoseconds. Also the simulations on the largest systems were performed in gas phase, mimicking the properties in a poor solvent, but leaving open questions of properties in good solvents, for example the effect of $p \mathrm{H}$.

We have also performed simulations in explicit water and ions for PAMAM dendrimers up to generation 8 , addressing the $p \mathrm{H}$ responsive behavior of the PAMAM dendrimers and complexation of DNA with dendrimers. ${ }^{7-10}$ In addition Han et al. ${ }^{11}$ reported various structural properties of PAMAM dendrimer up to G7 in explicit water. More recently Opitz and Wagner ${ }^{12}$ reported a detailed study of PAMAM dendrimer of generations G3 to G6 in methanol solution using fully atomistic simulation. We have also reported simulation studies of polypropyletherimine dendrimer in explicit solvent to validate the results from the small angle $\mathrm{x}$-ray experiment (SAXS). ${ }^{13}$ Again these studies were limited to lower generation dendrimer (up to G6 and G7) and short time scales (up to a few nanoseconds). Recently Lee et al. ${ }^{14}$ reported long time scale molecular dynamics (MD) simulation of G5 PAMAM dendrimer in ethanol/methanol solution and calculated the size of the dendrimer under various sol- 
vent conditions. All these studies have provided increased insight into some structural and dynamical properties of dendrimer systems, but there remain controversies regarding the distribution of the terminal end groups within the dendrimers and their location on the periphery of the molecule as well as $p \mathrm{H}$ responsive swelling of PAMAM dendrimers. Also all these studies were limited to the structural and dynamical properties of the dendrimer at a single molecule level.

No atomistic simulations have considered a concentrated solution of dendrimer where dendrimer-dendrimer interactions could be important. This is an important gap in understanding since many applications might depend on the nature of effective interaction between the dendrimers. Again atomistic level studies for such systems are computationally demanding.

These difficulties summarized above demand an atomistically informed coarse-grained description of the PAMAM dendrimers. Several coarse-grained descriptions of the dendrimers have been proposed. ${ }^{15-34}$ However, most previous studies have used very simple bead model to obtain qualitative features with no attempt to develop a coarse-graining scheme that would reproduce the structures and properties obtained from fully atomistic simulations. An objective of the present work is to address this lacuna, by deriving a mesoscale description based on fitting the underlying fully atomistic description. Recently Gurtovenko et al. ${ }^{32}$ proposed a coarse-grained model that can handle both the charged and uncharged dendrimer and were able to reproduce the experimental radii of gyration of PAMAM dendrimer as a function of generation for lower generations. Lee and Larson ${ }^{35}$ extended the Gurtovenko coarse-graining scheme to account for the presence of the different kinds of monomers characteristic of real dendrimers. However, their study was limited to the G3 and G5 PAMAM dendrimer in the context of dendrimers interacting with lipid bilayer.

We report here a general approach to develop coarsegrained models for dendrimers, which significantly reduces the degrees of freedom while retaining the accuracy of the fully atomistic model. We adopted a scheme shown schematically in Fig. 1, in which each monomer segment is replaced by two beads. Each bead represents half a generation and sits at the nitrogen position. This scheme is similar in spirit to the model of Lee and Larson, ${ }^{35}$ who also used two beads on the nitrogen sites. However, here we develop a general scheme and a continuous configurational biased method with pseudo-TX (CCBTX) atom (Sec. II), which allowed us to study the structural aspects of the dendrimer up to limiting generations which was lacking so far. To derive the force field parameters for our coarse-grained atoms, we used the fully atomistic simulation of G6 dendrimer in gas phase. The developed coarse-grained model will be made available freely for further use by the community.

The paper is organized as follows: Section II describes in detail the construction of the coarse-grained model and simulation details, Sec. III gives a summary of the results obtained from our MD simulations, and Sec. IV provides the conclusion.

\section{MODEL SYSTEMS CONSTRUCTIONS AND METHOD USED IN SIMULATIONS}

We generated the initial three-dimensional mesoscale structures of PAMAM dendrimers up to generation 11 in two ways: First we generate the fully atomistic details model using continuous configurational Boltzmann biased (CCBB) direct Monte Carlo (MC) method ${ }^{36,37}$ and then mapped them onto the coarse-grained model according to the scheme given in Fig. 1. This approach allowed building up to generation 11 mesoscale model only because bad contacts caused the fully atomistic model to fail beyond generation 11. Table I gives a comparison of the number of atoms in the atomistic model as well as in the coarse-grained model and we see a ten-time reduction in the number of atoms in the coarse-grained model.

Second we generated the coarse-grained structure using the CCBB method as outlined in Sec. II A. In both cases the initial structures were subjected to conjugate gradient optimization to obtain lower energy configurations. We further annealed the minimized structures through a cycle of quench anneal procedures at temperatures up to $600 \mathrm{~K}$ and cooling back to $300 \mathrm{~K}$. This final structure was then used for $300 \mathrm{~K}$ MD simulations to obtain equilibrium structures and properties.

\section{A. CCBTX method and construction of dendrimers}

In order to predict the polymer properties it is necessary to determine an equilibrium ensemble of conformations highly populated at the temperature and/or pressure of interest. An efficient method for predicting these conformations is MC sampling. The CCBTX method, an improved MC method as described below, was developed to serve this purpose. Here, we have applied it to generate initial structures of PAMAM dendrimers.

The continuous configurational biased $(\mathrm{CCB})$ direct $\mathrm{MC}$ method is developed on the basis of independent rotational sampling (IRS) method. In IRS method, torsions in the polymer chains are sampled using a weighting function based on the Boltzmann factor of the torsion energy. For IRS, the normalized torsion weighting function (TWF), $W_{\text {IRS }}$ is defined as

$$
W_{\mathrm{IRS}}(\phi)=\frac{g_{\mathrm{IRS}}(\phi)}{z_{\mathrm{IRS}}},
$$

where

$$
\begin{aligned}
& z_{\mathrm{IRS}}=\int_{0}^{2 \pi} g_{\mathrm{IRS}}(\phi) d \phi, \\
& g_{\mathrm{IRS}}(\phi)=\exp \left[-\beta E_{t}(\phi)\right] .
\end{aligned}
$$

However, IRS does not permit exclusion between nonbonding atoms having high spatial overlaps, leading to many configurations with impossibly high energy.

In order to remedy this problem, nonbonding interactions in the vicinity of the growing chain end and within a cutoff sphere are introduced into the TWF calculation. Thus, 

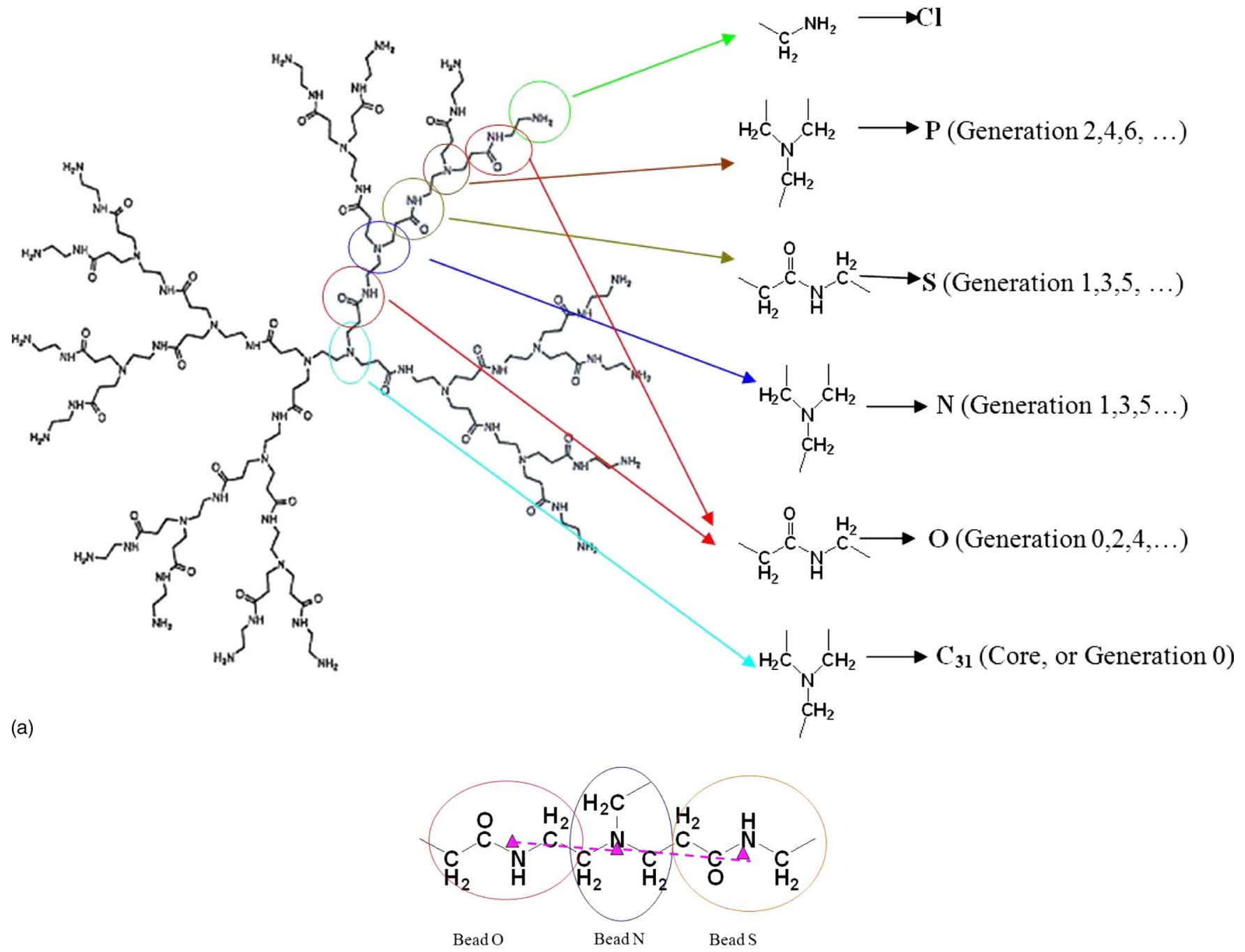

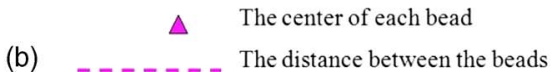

FIG. 1. (Color online) (a) Mapping of dendrimer PAMAM segments into coarse-grained beads. Each monomer is represented by two beads N1 or N2. N1 is represented by $\mathrm{O}$ or $\mathrm{S}, \mathrm{N} 2$ is represented by $\mathrm{N}$ or P. Note that N1-N2 need not be equal to N2-N1. In order to keep track of this difference we used O_3 and S_3 atom types for $\mathrm{N} 1$ and $\mathrm{N} \_3$ and $\mathrm{P} \_3$ atom types for N2). Other than the definition of bond distances the force field attributes of $\mathrm{N} \_3$ and $\mathrm{P} \_3$ (O_3 and S_3) are identical. (b) The same segment in PAMAM has different bond lengths and we apply special protocol to label each segment. The distance between the center of beads $\mathrm{O}$ and $\mathrm{N}$ is different from the distance between the center of beads $\mathrm{N}$ and $\mathrm{S}$, although beads $\mathrm{O}$ and $\mathrm{S}$ represent exactly the same segment $(-\mathrm{CH} 2-\mathrm{CO}-\mathrm{NH}-\mathrm{CH} 2)$. The reason is that the segment is not symmetrical in two directions. Beads $\mathrm{O}$ and $\mathrm{S}$ are labeled as $\mathrm{N} 1$ and bead $\mathrm{N}$ is labeled as $\mathrm{N} 2$. So the N1-N2 (O-N) distance (within the same generation) is different from the N2-N1(N-S) distance (between the two successive generation).

the Boltzmann factor, $W_{\mathrm{CCB}}$, for the nonbonding energy inside the cutoff sphere of radius $R_{C}$, is introduced into the TWF,

$W_{\mathrm{CCB}}\left(\phi_{i} ; \phi_{1}, \ldots, \phi_{i-1}\right)=\frac{g_{\mathrm{CCB}}\left(\phi_{i} ; \phi_{1}, \ldots, \phi_{i-1}\right)}{z_{\mathrm{CCB}}\left(\phi_{1}, \ldots, \phi_{i-1}\right)}$,

where

$z_{\mathrm{CCB}}\left(\phi_{1}, \ldots, \phi_{i-1}\right)=\int_{0}^{2 \pi} g_{\mathrm{CCB}}\left(\phi_{i} ; \phi_{1}, \ldots, \phi_{i-1}\right) d \phi_{i}$,

$g_{\mathrm{CCB}}\left(\phi_{i} ; \phi_{1}, \ldots, \phi_{i-1}\right)$

$$
=g_{\mathrm{IRS}}\left(\phi_{i}\right) \exp \left[-\beta \sum_{j, k} \Theta\left(R_{C}-r_{j k}\right) E_{L J}\left(r_{j k}\right)\right] .
$$

Here atom $j$ belongs to the growing chain end group and
TABLE I. Comparison of the number of atoms for various generation PAMAM dendrimers for fully atomistic and coarse-grained models. The degrees of freedom are reduced by a factor of 10 .

\begin{tabular}{crr}
\hline \hline Generation & Number of atoms (atomistic) & Number of atoms (CG) \\
\hline 0 & 84 & 10 \\
1 & 228 & 26 \\
2 & 516 & 58 \\
3 & 1092 & 122 \\
4 & 2244 & 250 \\
5 & 4548 & 506 \\
6 & 9156 & 1018 \\
7 & 18372 & 2042 \\
8 & 36804 & 4090 \\
9 & 73668 & 8186 \\
10 & 147396 & 16378 \\
11 & 294852 & 32762 \\
\hline
\end{tabular}


atom $k$ is one of the atoms in the grown polymer chains. Moreover $\Theta(R)$ is the Heavside step function, namely, $\Theta(R)=0$ when $R<0$ and $\Theta(R)=1$ when $R \geq 0$.

Prior to each step of chain sampling, the torsion energy and nonbonding energy within the cutoff radius of growing end is calculated for a fixed number of grid points equally separated from 0 to $2 \pi$ and $W_{\text {IRS }}$ was evaluated. $P_{\mathrm{IRS}}(\phi)$ is defined as the auxiliary distribution and as

$$
P_{\mathrm{IRS}}(\phi)=\int_{0}^{\phi} W_{\mathrm{IRS}}\left(\phi^{\prime}\right) d \phi^{\prime} .
$$

A random number $\xi$, uniformly distributed in the interval $[0,1)$, is drawn and the torsion angle is obtained by requiring

$$
P_{\mathrm{IRS}}\left(\phi_{i}\right)=\int_{0}^{\phi} W_{\mathrm{CCB}}(\phi) d \phi .
$$

We extended the CCB method described above to generate condensed state of multichain amorphous polymer and dendrimer systems in periodic cells. The extended method denoted as CCBTX was also extended to generate high quality dense polymer/dendrimer systems with any detailed atomistic architecture (the previous CCB method focused on simulation of united atomistic model of polyethylene). CCBTX has been applied to generate structures for PAMAM dendrimer of generations 1-11, supramolecular assemblies of spherical and cylindrical giant liquid crystalline structure of Li et al. ${ }^{38}$ and various other polymers. ${ }^{39,40}$

The CCB method grows the polymer/dendrimer system segment by segment in a defined sequence. The assumption is that while we sample the torsion of an early segment in a chain (or dendrimer), we ignore the locations of atoms in future segments. This is not a problem for MC sampling of isolated chains because sufficient space is generally accessible for future segments. However for dense amorphous polymer or dendrimer systems, a majority of the chains may not be able to be completed because of severe close contacts. "Future scan" strategies do remedy this problem efficiently for multiple chains or dendrimer system because of uncertainty in the positions of the atoms of other chains or branches.

When sampling $i$ th segment in dense multiple chains polymer or dendrimer, we do not know the positions of the atoms in the future segments. The future segments include not only the future segments in the same chain/branch of $i$ th segment, but also the future segments in the other chains/ branches. We solve this problem by placing one pseudo-TX atom (or multiple pseudo-TX atoms for dendrimers) at the end of each chain/branch to represent the future segments of that chain/branch. This extension of the CCB method using TX pseudoatoms in torsion sampling is denoted as the CCBTX method,

$$
\begin{aligned}
& W_{i}^{\mathrm{CCBTX}}\left(\phi_{i} ; \phi_{4}, \ldots, \phi_{i}\right)=\frac{g_{i}^{\operatorname{CCBTX}}\left(\phi_{i} ; \phi_{4}, \ldots, \phi_{i-1}\right)}{z_{i}^{\operatorname{CCBTX}}\left(\phi_{4}, \ldots, \phi_{i-1}\right)}, \\
& z_{i}^{\operatorname{CCBTX}}\left(\phi_{4}, \ldots, \phi_{i-1}\right)=\int_{0}^{2 \pi} g_{i}^{\operatorname{CCBTX}}\left(\phi_{i} ; \phi_{4}, \ldots, \phi_{i-1}\right) d \phi_{i}, \\
& g_{i}^{\operatorname{CCBTX}}\left(\phi_{i} ; \phi_{4}, \ldots, \phi_{i-1}\right) \\
& =g_{\text {IRS }}\left(\phi_{i}\right) \exp \left[-\beta \sum_{j=1}^{i-4} \Theta\left(R_{C}-r_{i j}\right) E_{L J}\left(r_{i j}\right)\right. \\
& \left.\quad \beta \sum_{k=1}^{\operatorname{NumTX}} \Theta\left(R_{C}-r_{i k}\right) E_{L J}\left(r_{i k}\right)\right] .
\end{aligned}
$$

\section{B. Coarse-grained force field}

To develop the coarse-grain force field (CG-FF), we used a procedure similar to that used by Molinero and Goddard $^{41,42}$ to obtain a CG-FF for describing oligosaccharides and their water mixtures, which was applied successfully to larger scale diffusional processes. ${ }^{43,44}$ Thus we based our coarse-grain internal degrees of freedom on a 600 ps 300 $\mathrm{K}$ fully atomistic MD simulation of generation 6 PAMAM dendrimer in gas phase.

For bond stretch and angle bending in the coarse-grain bead model we have used classical harmonic potentials, where interaction centers are chosen to be at N1 and N2 positions of monomers,

$$
E_{\text {bond }}=\frac{1}{2} k_{b}\left(b-b_{0}\right)^{2},
$$

and

$$
E_{\text {bend }}=\frac{1}{2} k_{\theta}\left(\theta-\theta_{0}\right)^{2},
$$

where $k_{b}$ and $k_{\theta}$ are the force constants for bond stretch and angle bending respectively; $b_{0}$ and $\theta_{0}$ are the corresponding equilibrium bond length and angle values.

Since each bead is to be placed at either N1 or N2 sites of each monomer, we collected the distribution of N1-N2 and N2-N1 distances from the simulations. Note that N1-N2 need not be equal to N2-N1. In order to keep track of this difference we used O_3 and S_3 atom types for N1 and N_3 and $\mathrm{P}_{3} 3$ atom types for $\mathrm{N} 2$ [see Fig. 1(a)]. Other than the definition of bond distances the force field attributes of N_3 and $\mathrm{P} \_3\left(\mathrm{O} \_3\right.$ and $\left.\mathrm{S} \_3\right)$ are identical. Then the equilibrium distances $b_{0 O \_3-N \_3}$ and $b_{0 N \_3-S \_3}$ (equivalently $b_{0 \text { S_3-P_3 }}$ and $\left.b_{0 \mathrm{P} \_3-\mathrm{O} \_3}\right)$ were chosen to match the average value. Similarly to obtain the angle bending parameters, we have calculated the O_3-N_3-S_3 and N_3-S_3-P_3 angle distributions and the averages from the atomistic simulations, and used this to

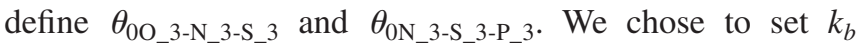
$=70 \mathrm{kcal} / \mathrm{mol} \mathrm{A}$ and $k_{\theta}=10 \mathrm{kcal} / \mathrm{mol} \mathrm{rad}$, which are $1 / 10 \mathrm{th}$ of the values used in Dreiding force field (see Table II for further details).

As shown in Fig. 1(b), The distance between the center of beads $\mathrm{O}$ and $\mathrm{N}$ is different from the distance between the center of beads $\mathrm{N}$ and $\mathrm{S}$, although beads $\mathrm{O}$ and $\mathrm{S}$ represent 
TABLE II. Van der Waals interaction parameters for Lennard Jones 12-6 interaction.

\begin{tabular}{lcc}
\hline \hline Atom & $\begin{array}{c}R_{o} \\
\AA\end{array}$ & $\begin{array}{c}D_{o} \\
(\mathrm{kcal} / \mathrm{mol})\end{array}$ \\
\hline C_31 & 4.5 & 0.25 \\
O_3 & 5.0 & 0.40 \\
N_3 & 4.7 & 0.30 \\
S_3 & 5.0 & 0.40 \\
P_3 & 4.7 & 0.30 \\
Cl & 4.7 & 0.30
\end{tabular}

Bond stretch force field parameters for harmonic interactions $\frac{1}{2} k_{b}\left(b-b_{0}\right)^{2}$

\begin{tabular}{lcc} 
Bond & $\begin{array}{c}k_{b} \\
\left(\mathrm{kcal} / \mathrm{mol} / \mathrm{A}^{2}\right)\end{array}$ & $\begin{array}{c}b_{o} \\
(\AA)\end{array}$ \\
\hline C_31 C_31 & 70.0 & 3.8 \\
C_31 S_3 & 70.0 & 4.5 \\
O_3 C_31 & 70.0 & 4.5 \\
O_3 N_3 & 70.0 & 3.0 \\
P_3 O_3 & 70.0 & 4.7 \\
S_3 N_3 & 70.0 & 4.7 \\
S_3 P_3 & 70.0 & 3.0 \\
S_3 S_3 & 70.0 & 3.0 \\
Cl-O_3 & 70.0 & 3.5 \\
C1_S_3 & 70.0 & 3.0
\end{tabular}

Angle bending force field parameters for harmonic interactions: $\frac{1}{2} k_{\theta}\left(\theta-\theta_{o}\right)^{2}$

\begin{tabular}{lcc} 
& $\begin{array}{c}k_{\theta} \\
\text { Angle }\end{array}$ & $\begin{array}{c}\theta_{o} \\
\left(\mathrm{kcal} / \mathrm{mol} \mathrm{rad}^{2}\right)\end{array}$ \\
\hline X C_31 X & 10.0 & 109.47 \\
X C_3X & 10.0 & 109.47 \\
X N_3 X & 10.0 & 106.70 \\
X O_3 X & 10.0 & 104.51 \\
X P_3 X & 10.0 & 93.30 \\
X S_3 X & 10.0 & 92.10 \\
\hline \hline
\end{tabular}

exactly the same segment $(-\mathrm{CH} 2-\mathrm{CO}-\mathrm{NH}-\mathrm{CH} 2)$. The reason is that the segment is not symmetrical in two directions. The bond distance of $\mathrm{O} \_3-\mathrm{N} \_3$ is $3.0 \mathrm{~A}$ and it is $4.7 \mathrm{~A}$ for S_3-N_3, as shown in Table II. This is a smart way to label the same chemistry with different beads so that we could capture difference in bond length difference for first and second halves of each generation. We chose the charge of the beads as neutral since each half generation monomers used in the atomistic representation were neutral.

For the nonbond (van der Waals) interactions we have used the standard 12-6 potential in the following form:

$$
E(r)=D_{e}\left[\left(R_{0} / R\right)^{12}-2\left(R_{0} / R\right)^{6}\right] .
$$

We fitted the interactions at the monopole level. That is we calculated the pair distribution $R_{i j}$ but excluding near neighbor (1-2) and next near neighbor (1-3) interactions. Thus the next-next-neighbor (1-4) interactions are handled fully within the nonbond terms, with no explicit torsion or inversion terms. We refined these nonbond parameters, adjusting the parameters to fit the overall distribution and approximating the attractive value to $\sqrt{D_{0} r_{o}^{3}}$ and the repulsive value to $\sqrt{D_{0} r_{o}^{6}}$ for the collection of atoms making up the beads. The

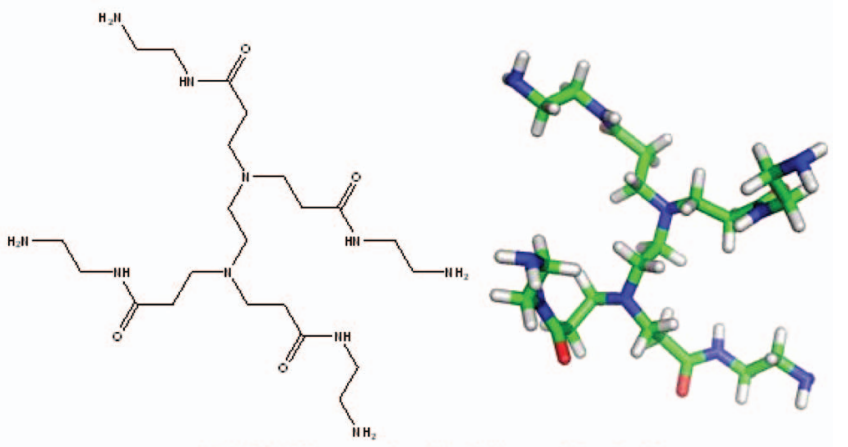

(a) PAMAM generation 0 in full atomistic details
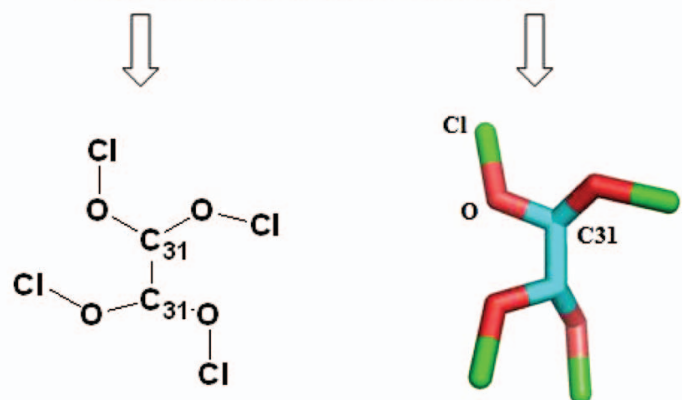

(b) PAMAM generation 0 in coarse-grained model

FIG. 2. (Color) (a) Generation 0 dendrimer in full atomistic details (top) and (b) in the coarse-grained description (bottom).

parameters used for this coarse-grained PAMAM dendrimer force field (denoted as CG-D) are given in Table II.

Note that the atom type used in the coarse-grained model (C_31, O_3, N_3, P_3, S_3 , and Cl) does not have any correspondence with element names and atomic detail force field types. They are introduced to distinguish the beads belonging to two successive generations and to address the intergeneration and between intrageneration bond distance and bond force constant differences. As the bead force field types $\mathrm{N} \_3$ and $\mathrm{P} \_3$ are identical, $\mathrm{O} \_3$ and $\mathrm{S} \_3$ are also identical bead force field types. Figure 2 shows the mapping of atomistic to coarse-grained model for G0 dendrimer.

\section{MD simulations}

We carried out MD simulations at room temperature for generations 1-11. These simulations employed the CG-D force field described above to describe the interatomic interactions. To evaluate the nonbonded van der Walls interactions, we employed the cell multipole method, ${ }^{45}$ which includes the interactions with all atoms (no cutoffs), using multipole expansions. These calculations used up to quadrupole expansions in the far field and local potential. The bounding box was adjusted to have an average of 6-10 particles per leaf cell.

All calculations reported here were carried out with the MPSIM program. ${ }^{46}$ MD simulations employed an integration step of 2-5 fs to maintain conservation of Hamiltonian. The simulations were conducted at $T=300 \mathrm{~K}$, and for generations 3-8 we used $200 \mathrm{ps}$ for equilibration and several ns for the data collection. For generations 10 and 11 we used 200 ps for equilibration and 2-3 ns for the data collection. The equilibration process was monitored by measuring the total 


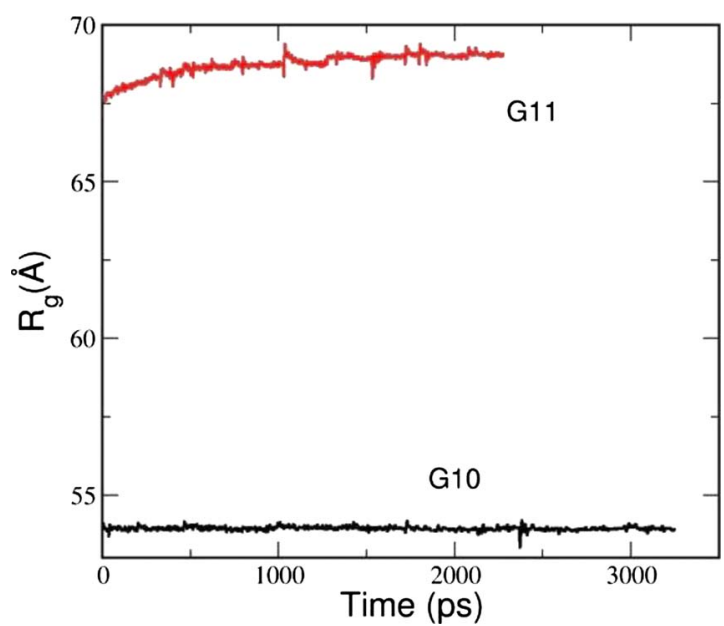

FIG. 3. (Color online) Time evolution of the radius of gyration $\left(R_{g}\right)$ of the PAMAM dendrimers, generation 10 (3 ns) and generation $11(2 \mathrm{~ns})$ during simulation.

energy as well as the instantaneous radius of gyration of the dendrimer. As shown in Fig. 3, the variation of the radius of gyration as a function of time for generation $10(3 \mathrm{~ns})$ and generation $11(2 \mathrm{~ns})$, the simulations are long enough to obtain reliable structural and thermodynamic properties. Furthermore for higher generations, the branched topology of the dendrimers inhibits major conformational excursions that might be observed in linear polymers in long time simulations, thus the length of simulations used in this study is long enough to determine physical and structural properties of high generation dendrimers. We saved the configurations at $0.5 \mathrm{ps}$ interval and used these configurations to determine the average properties at $T=300 \mathrm{~K}$. Section III presents the simulation results.

\section{RESULTS}

\section{A. Size and shape}

A good test of the coarse-grained model is to compare the dendrimer shape and size with those obtained from the previous atomistic simulation as well the data obtained from various small angle x-ray scattering (SAXS) and small angle neutron scattering (SANS) experiments. A quantitative estimate of the dendrimer shape and size can be described by the mean-square radius of gyration $\left\langle R_{g}^{2}\right\rangle$ and shape tensor of the individual chain. For a chain with $N$ monomers the meansquare radius of gyration is given by

$$
\left\langle R_{g}^{2}\right\rangle=\frac{1}{\sum_{i=1}^{N} m_{i}}\left\langle\left[\sum_{i=1}^{N} m_{i}\left|r_{i}-R\right|^{2}\right]\right\rangle,
$$

where $R$ is the center of mass of the chain. $m_{i}$ is the mass of the $i$ th monomer.

Figure 4(a) and Table III show the radius of gyration $R_{g}$ as a function of generation. Here, we see good agreement between our calculated $R_{g}$ with those obtained form our
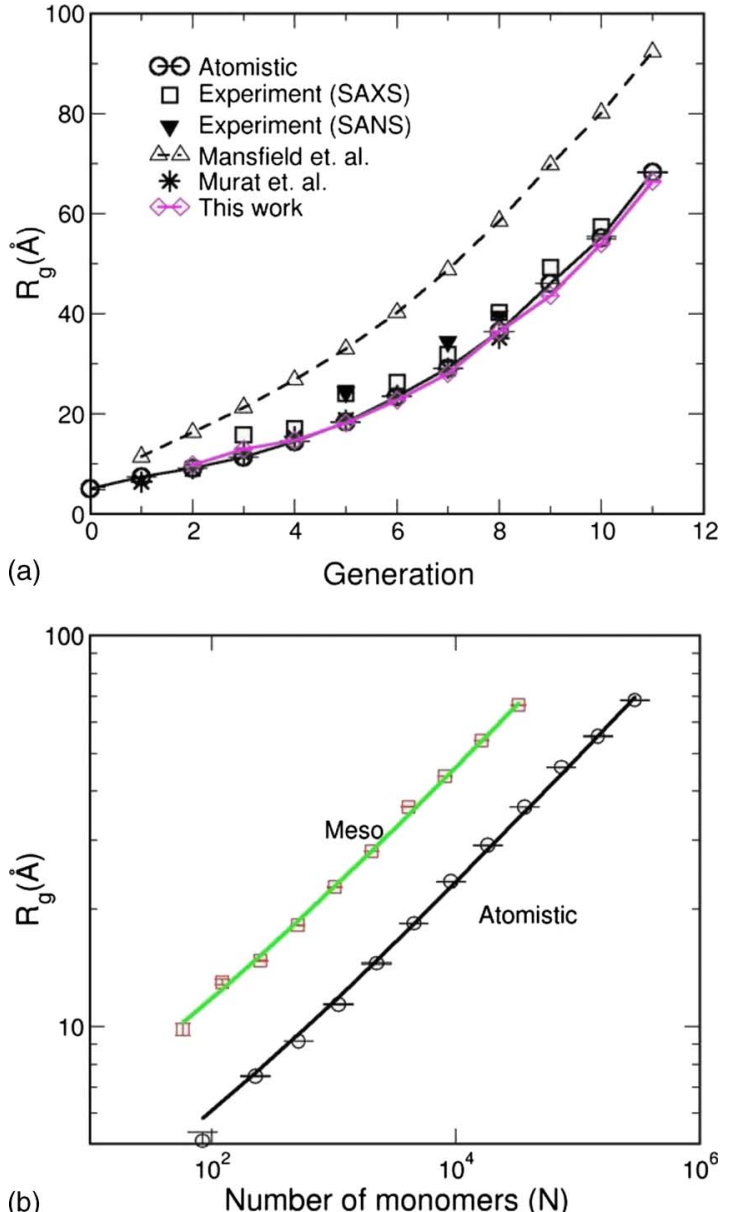

FIG. 4. (Color online) (a) The average radius of gyration $R_{g}$ of dendrimers as a function of generation. Our values were averaged from the snapshots every $0.5 \mathrm{ps}$ from the equilibrium MD trajectory (i.e., after equilibration run). For comparison we have also included the results obtained from our previous atomistic simulation. The data obtained from SAXS and SANS experiments are also shown. (b) A log-log plot of calculated $R_{g}$ from our simulations as a function of the number of atoms $(n)$ in the dendrimer. The solid line shows the fit, $R_{g} \sim N^{0.32}$.

recent all atom MD simulation in gas phase. ${ }^{6}$ For comparison we have also included the data from the recent SANS (Refs. 47 and 48) and SAXS experiments. ${ }^{49}$ Size obtained from our coarse-grained model matches quite well with the SAXS and SANS data as well. Another interesting feature is that $R_{g}$ has a small increase with generation for smaller generations and then a sudden increase in $R_{g}$ for higher generation. Similar behavior is also observed in other theoretical model $^{50}$ and simulation results. ${ }^{18,25}$ In Fig. 4(b) We have plotted the radius of gyration $R_{g}$ as a function of number of monomers in the dendrimer and we see that $R_{g} \sim N^{0.32}$. The scaling exponent is in excellent agreement with the available theoretical results and other simulation results. This indicates a very compact structure indicative of their behavior in poor solvent. Our present results along with our previous all atom simulation in gas phase ${ }^{6}$ as well as in explicit water ${ }^{51}$ conclusively demonstrate a universal scaling law which the governs the size dependence on the molecular weight of the PAMAM dendrimer up to limiting generations.

The shape tensor describing the mass distribution is given by 
TABLE III. Radius of gyration $\left(R_{g}\right)(\AA)$ as a function of generation for PAMAM. Our values were averaged from snapshots every $0.5 \mathrm{ps}$ in the dynamics (after equilibration). SAXS results are from Ref. 54. SANS results for generations 5 and 8 are from Ref. 47 and for generation 7 are from Ref. 55. Mansfield and Jeong (Ref. 18) used MC simulations on a diamond lattice dendrimer model described by seven step spacers using spacers of step size $b=3^{1 / 2}$. Murat and Grest (Ref. 25) used Brownian dynamics simulations on a bead model dendrimers with seven step spacers on with average bond length $b=0.97$.

\begin{tabular}{|c|c|c|c|c|c|c|}
\hline \multirow[b]{2}{*}{ Generation } & \multirow{2}{*}{$\begin{array}{c}\text { This work } \\
R_{g}\end{array}$} & \multirow{2}{*}{$\begin{array}{c}\text { Atomistic } \\
\text { Ref. } 6 \\
R_{g}\end{array}$} & \multicolumn{2}{|c|}{ Experiment } & \multirow{2}{*}{$\begin{array}{c}\text { Mansfield } \\
R_{g}\end{array}$} & \multirow{2}{*}{$\begin{array}{c}\text { Murat } \\
R_{g}\end{array}$} \\
\hline & & & SAXS & SANS & & \\
\hline 0 & & 4.93 & & & & \\
\hline 1 & & 7.46 & & & 7.63 & 4.27 \\
\hline 2 & 9.83 & 9.17 & & & 10.88 & 6.01 \\
\hline 3 & 13.0 & 11.23 & 15.8 & & 14.2 & 7.96 \\
\hline 4 & 14.72 & 14.5 & 17.1 & & 17.87 & 10.27 \\
\hline 5 & 18.15 & 18.34 & 24.1 & 24.3 & 21.99 & 12.49 \\
\hline 6 & 22.73 & 22.4 & 26.3 & & 26.82 & 15.79 \\
\hline 7 & 28.05 & 29.09 & 31.9 & 34.4 & 32.48 & 19.37 \\
\hline 8 & 36.48 & 36.42 & 40.3 & 39.5 & 39.04 & 20.41 \\
\hline 9 & 43.63 & 46.03 & 49.2 & & 46.47 & \\
\hline 10 & 53.92 & 55.19 & 57.4 & & 53.42 & \\
\hline 11 & 66.38 & 68.25 & & & 61.51 & \\
\hline
\end{tabular}

$$
G_{m n}=\frac{1}{N}\left[\sum_{i}^{N}\left(r_{m i}-R_{m}\right)\left(r_{n i}-R_{n}\right)\right], \quad m, n=x, y, z .
$$

The three eigenvalues of $\mathbf{G}$ are denoted by $g_{1}, g_{2}$, and $g_{3}$ (in descending order). $\left\langle R_{g}^{2}\right\rangle$ is the sum of these three eigenvalues. The ratio of these three eigenvalues determines the shape of the dendrimer. A better definition for asphericity introduced by Rudnick and Gaspari ${ }^{52}$ and frequently used in literature is defined as

$$
\delta=1-3 \frac{\left\langle I_{2}\right\rangle}{\left\langle I_{1}^{2}\right\rangle},
$$

where $I_{i}$ are the respective invariant of the gyration tensor and is given by $I_{1}=g_{1}+g_{2}+g_{3} I_{2}=g_{1} g_{2}+g_{2} g_{3}+g_{3} g_{1}$ and $I_{3}$ $=g_{1} g_{2} g_{3}$.

The shape of the dendrimer can be assessed from the average values of the ratio of three principal moments of inertia of the molecules. In figure we plot the average ratios for different generation dendrimers. We see that over the entire generation range we have simulated $g_{1} / g_{2}$ is in the range $1.0-1.3$ and $g_{1} / g_{3}$ is in the range $1.1-1.7$. This means that these dendrimer are almost spherical in shape. In Fig. 5 we show the asphericities of dendrimers as function of generation. For comparison we have also included the results from our previous atomistic simulation.

We find a good quantitative agreement between the two up to G6. For G7-G11 our coarse-grained model predict less asphericities compared to the atomistic model. The reason might be that fact that our coarse-grained model are better equilibrated over long runs (several nanoseconds) compared to the few hundreds of picosecond runs for the larger generation atomistic model. To validate this claim we need longer runs for the gas phase atomistic simulation of the higher generation dendrimers. However overall our atomistically informed coarse-grained model can reproduce the size and shape of the PAMAM dendrimer very reliably. The asphericities of dendrimers shown in Fig. 5 also reveal a strongly compact spherical structure. From the instantaneous snapshots (shown in Fig. 6) also we see that their shape does not deviate much from a perfect sphere.

\section{B. Radial monomer density profile}

Another test for the validity of the mesomodel is to investigate the internal structure of dendrimer and compare with the available atomistic simulation results and experimentally obtained density distribution. The average radial monomer density $\rho(r)$ can be defined by counting the number $N(r)$ of monomers whose centers of mass are located within the spherical shell of radius $r$ and thickness $\Delta r$. Hence, the integration over $r$ yields the total number of monomers as

$$
N(r)=4 \pi \int_{0}^{\infty} r^{2} \rho(r) d r
$$

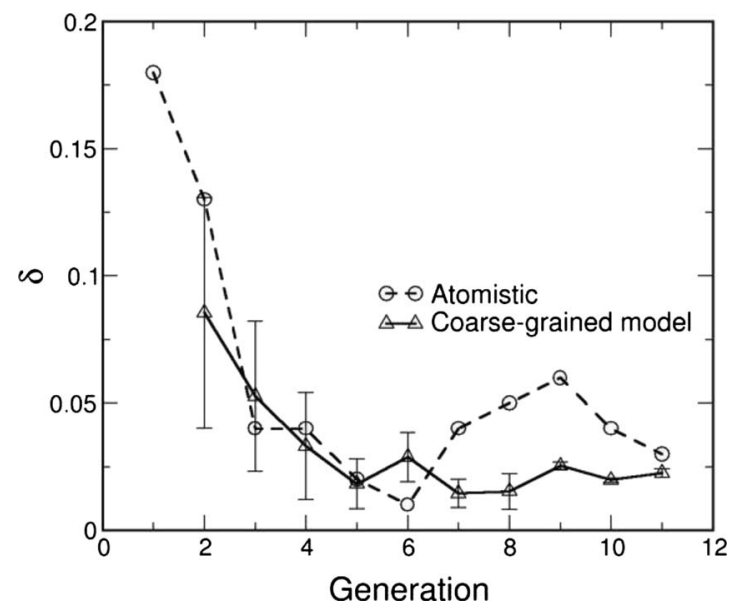

FIG. 5. The asphericity parameter, $\delta$, as a function of generation. The values plotted were averaged using the snapshots every $0.5 \mathrm{ps}$ from the equilibrium MD trajectory. The solid line is only to guide the eye. 


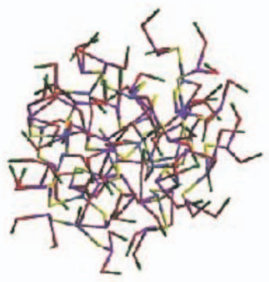

G4

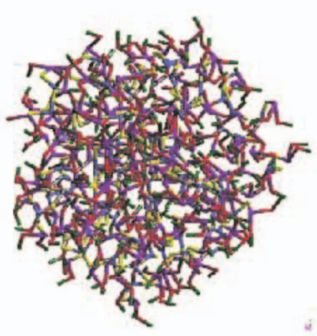

G6

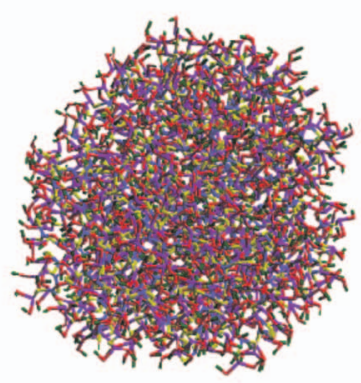

G8

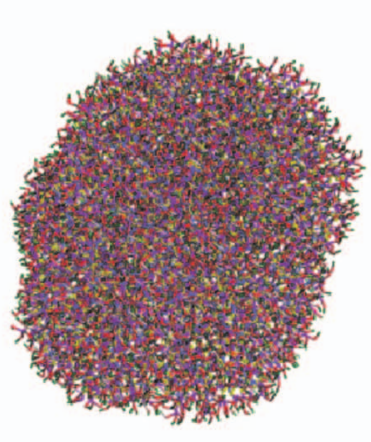

G10

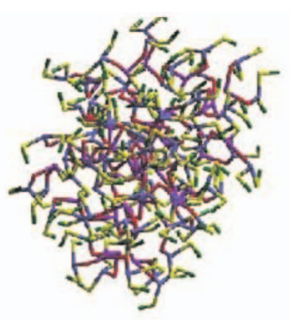

G5

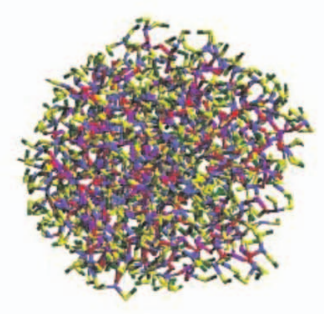

G7

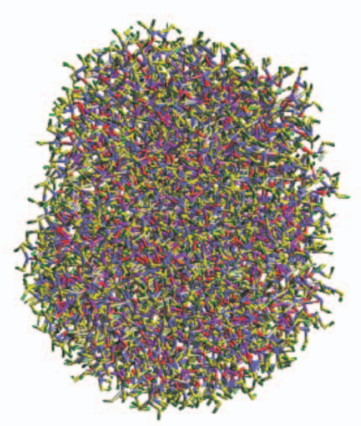

G9

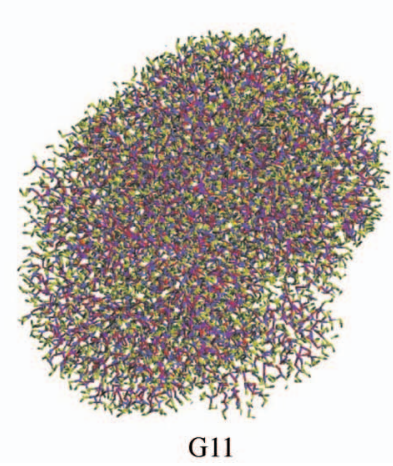

G11
FIG. 6. (Color) Instantaneous snapshots of G1-G11 PAMAM dendrimers after long MD simulations at $T=300 \mathrm{~K}$. All figures are to the same scale. Here, the blue atoms are nitrogen, the red atoms are oxygen, the yellow atoms are phosphorus, and the green atoms are chlorine.

In Fig. 7(a) we have plotted the radial monomer density for different generation PAMAM dendrimers. The contributions of the monomers belonging to consecutive generations are also shown. It shows a minimum at the core and gradually increases with a maximum at some distance from the core which roughly corresponds to its radius of gyration. After this gradual increase there is a region of almost constant density and the monomer density in this constant density regime is increases with the generation. The region of the constant density zone increases with the increase in den- drimer generation. Beyond that it decays monotonically. This density profile is very different from those obtained from the self-consistent mean field model of Boris and Rubinstein, ${ }^{53}$ which predicts the highest density at the core, followed by a monotonic decay to the edge of the molecule. The density profiles for various subgenerations demonstrate a high degree of back folding. Similar behavior is also seen in our previous all atom MD simulations up to generation $11 .{ }^{6}$ To have a better comparison with the fully atomistic monomer density profile we have plotted the coarse-grained density profile and fully atomistic density profile in the same plot as in Fig. 7(b) for G4 and G8. In the last Fig. 7(a) we compare the radial monomer density profiles for each generation, from G1 to G11. One interesting feature is that in the near vicinity of the core the monomer density increases with the increasing generation in contrast to the fully atomistic case where opposite behavior was observed. Also we do not find a local density minimum near core of the molecule for smaller generation dendrimer as was seen in the atomistic simulation. This could be the effect of the level of coarse graining we have done. In accordance with atomistic simulation results our coarse-grained model also reproduces the region of almost constant density and the constant density regime increases with the generation. The extent of the constant density zone increases gradually for higher generations. The fact that the density in the plateau region increases with the dendrimer generation has been used by Götze and $\operatorname{Likos}^{30}$ to explain the deviation of the $1 / 3$ law in the scaling of $R_{g}$ with the number of monomers. However, in our simulation we still see $R_{g} \sim N^{1 / 3}$ over entire generation range. This demonstrates the reliability of our coarse-grained model to accurately describe the structure of real dendrimers. The reliability of our model to describe the dynamics of real dendrimer systems is yet to be demonstrated and is subject of a future study.

\section{SUMMARY AND CONCLUSION}

In summary we have developed a systematic coarsegraining scheme for PAMAM dendrimer based on the underlying atomistic model. This helps us to reduce the number of degrees of freedom by a factor of 10 . The resulting coarsegrained model reproduces the size, shape, and monomer density profile which are in quantitative agreement with the results from fully atomistic simulation as well as available experimental data. The model can be extended to include charge on the monomer to study the $p \mathrm{H}$ responsive behavior of PAMAM dendrimer in explicit water perhaps using mesowater as in the paper by Molinero and Goddard. ${ }^{42,43}$ The coarse-grained model can also be used to study the dynamics of PAMAM dendrimer over long time scales and is subject of future publication. Another interesting use of this model is to study the self-assembly behavior and ordering of PAMAM dendrimer.

\section{SUPPORTING INFORMATION AVAILABLE}

The $x y z$ coordinates for a snapshot of the trajectory for each dendrimer from generation 3 to generation 11 in asci format. $^{56}$ 

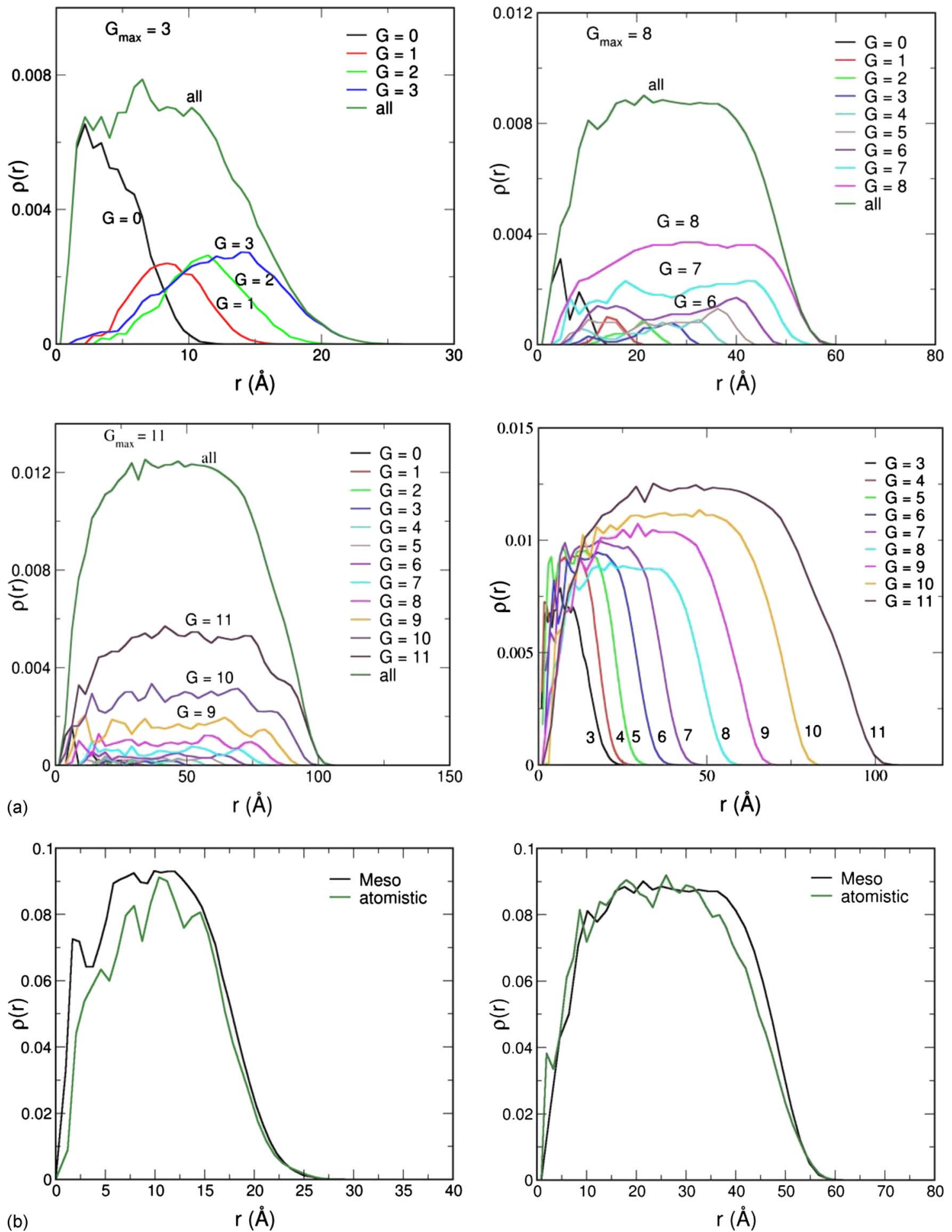

FIG. 7. (Color online) (a) Radial monomer densities for different generations of coarse-grained PAMAM dendrimers. Each figure is for a specific generation. The numbers shown were averaged from snapshots every $0.5 \mathrm{ps}$. The origin is at the center of mass. The last plot compares the total density profiles for all generations from G3 to G11. (b) Radial monomer densities for different generations of coarse-grained PAMAM dendrimers. Each figure is for a specific generation. The numbers shown were averaged from snapshots every $0.5 \mathrm{ps}$. The origin is at the center of mass. The last plot compares the total density profiles for all generations from G3 to G11.

\section{ACKNOWLEDGMENTS}

P.K.M. thanks DST, India for financial support. W.A.G. thanks NSF (CTS-0506951, CTS-0608889, CMMI-072870) for support.

${ }^{1}$ D. A. Tomalia, A. M. Naylor, and W. A. Goddard, Angew. Chem., Int. Ed. Engl. 29, 138 (1990).
${ }^{2}$ A. W. Bosman, H. M. Janssen, and E. W. Meijer, Chem. Rev. (Washington, D.C.) 99, 1665 (1999).

${ }^{3}$ C. C. Lee, J. A. MacKay, J. M. J. Frechet, and F. C. Szoka, Nat. Biotechnol. 23, 1517 (2005).

${ }^{4}$ S. Svenson and D. A. Tomalia, Adv. Drug Delivery Rev. 57, 2106 (2005).

${ }^{5}$ M. Ballauff and C. N. Likos, Angew. Chem. 43, 2998 (2004).

${ }^{6}$ P. K. Maiti, T. Cagin, G. F. Wang, and W. A. Goddard, Macromolecules 37, 6236 (2004). 
${ }^{7}$ P. K. Maiti, T. Cagin, S. T. Lin, and W. A. Goddard, Macromolecules 38 , 979 (2005).

${ }^{8}$ P. K. Maiti and W. A. Goddard, J. Phys. Chem. B 110, 25628 (2006).

${ }^{9}$ P. K. Maiti and R. Messina, Macromolecules 41, 5002 (2008).

${ }^{10}$ P. K. Maiti and B. Bagchi, Nano Lett. 6, 2478 (2006).

${ }^{11}$ M. Han, P. Q. Chen, and X. Z. Yang, Polymer 46, 3481 (2005).

${ }^{12}$ A. W. Opitz and N. J. Wagner, J. Polym. Sci., Part B: Polym. Phys. 44, 3062 (2006).

${ }^{13}$ C. Jana, G. Jayamurugan, R. Ganapathy, P. K. Maiti, N. Jayaraman, and A. K. Sood, J. Chem. Phys. 124, 204719 (2006).

${ }^{14}$ H. Lee, J. R. Baker, and R. G. Larson, J. Phys. Chem. B 110, 4014 (2006).

${ }^{15}$ R. L. Lescanec and M. Muthukumar, Macromolecules 23, 2280 (1990).

${ }^{16} \mathrm{P}$. Welch and M. Muthukumar, Macromolecules 31, 5892 (1998).

${ }^{17}$ P. Welch and M. Muthukumar, Macromolecules 33, 6159 (2000).

${ }^{18}$ M. L. Mansfield and M. Jeong, Macromolecules 35, 9794 (2002).

${ }^{19}$ M. L. Mansfield and L. I. Klushin, Macromolecules 26, 4262 (1993).

${ }^{20}$ M. L. Mansfield, Polymer 35, 1827 (1994).

${ }^{21}$ M. L. Mansfield, Polymer 37, 3835 (1996).

${ }^{22}$ M. L. Mansfield, Macromolecules 33, 8043 (2000).

${ }^{23}$ K. Karatasos, D. B. Adolf, and G. R. Davies, J. Chem. Phys. 115, 5310 (2001).

${ }^{24}$ N. Zacharopoulos and L. G. Economou, Macromolecules 35, 1814 (2002).

${ }^{25}$ M. Murat and G. S. Grest, Macromolecules 29, 1278 (1996).

${ }^{26}$ A. V. Lyulin, G. R. Davies, and D. B. Adolf, Macromolecules 33, 6899 (2000).

${ }^{27}$ A. V. Lyulin, G. R. Davies, and D. B. Adolf, Macromolecules 33, 3294 (2000).

${ }^{28}$ S. V. Lyulin, A. A. Darinskii, A. V. Lyulin, and M. A. J. Michels, Macromolecules 37, 4676 (2004).

${ }^{29}$ T. Terao and T. Nakayama, Macromolecules 37, 4686 (2004).

${ }^{30}$ I. O. Gotze and C. N. Likos, Macromolecules 36, 8189 (2003).

${ }^{31}$ N. W. Suek and M. H. Lamm, Macromolecules 39, 4247 (2006).

${ }^{32}$ A. A. Gurtovenko, S. V. Lyulin, M. Karttunen, and I. Vattulainen, J. Chem. Phys. 124, 094904 (2006).

${ }^{33}$ P. Carbone, A. Calabretta, M. Di Stefano, F. Negri, and K. Mullen, J. Phys. Chem. A 110, 2214 (2006).

${ }^{34}$ K. Karatasos, Macromolecules 41, 1025 (2008).
${ }^{35}$ H. Lee and R. G. Larson, J. Phys. Chem. B 110, 18204 (2006).

${ }^{36}$ J. Sadanobu and W. A. Goddard, J. Chem. Phys. 106, 6722 (1997).

${ }^{37}$ J. Sadanobu and W. A. Goddard, Fluid Phase Equilib. 144, 415 (1998).

${ }^{38}$ Y. Y. Li, S. T. Lin, and W. A. Goddard, J. Am. Chem. Soc. 126, 1872 (2004).

${ }^{39}$ S. T. Lin, S. S. Jang, T. Cagin, and W. A. Goddard, J. Phys. Chem. B 108, 10041 (2004).

${ }^{40}$ S. S. Jang, S. T. Lin, T. Cagin, V. Molinero, and W. A. Goddard, J. Phys. Chem. B 109, 10154 (2005).

${ }^{41}$ V. Molinero and W. A. Goddard, Nmr Spectroscopy and Computer Modeling of Carbohydrates: Recent Advances (American Chemical Society, Washington, DC, 2006), Vol. 930, pp. 271-284.

${ }^{42}$ V. Molinero and W. A. Goddard, J. Phys. Chem. B 108, 1414 (2004).

${ }^{43}$ V. Molinero and W. A. Goddard, Phys. Rev. Lett. 95, 045701 (2005).

${ }^{44}$ V. Molinero, T. Cagin, and W. A. Goddard, J. Phys. Chem. A 108, 3699 (2004).

${ }^{45}$ H. Q. Ding, N. Karasawa, and W. A. Goddard, J. Chem. Phys. 97, 4309 (1992).

${ }^{46}$ K. T. Lim, S. Brunett, M. Iotov, R. B. McClurg, N. Vaidehi, S. Dasgupta, S. Taylor, and W. A. Goddard, J. Comput. Chem. 18, 501 (1997).

${ }^{47}$ A. Topp, B. J. Bauer, D. A. Tomalia, and E. J. Amis, Macromolecules 32, 7232 (1999).

${ }^{48}$ A. Topp, B. J. Bauer, T. J. Prosa, R. Scherrenberg, and E. J. Amis, Macromolecules 32, 8923 (1999).

${ }^{49}$ S. Rathgeber, M. Monkenbusch, M. Kreitschmann, V. Urban, and A. Brulet, J. Chem. Phys. 117, 4047 (2002).

${ }^{50}$ P. Efthymiopoulos, M. Kosmas, C. Vlahos, and L. N. Gergidis, Macromolecules 40, 9164 (2007)

${ }^{51}$ P. K. Maiti and B. Bagchi, J. Phys. Chem. B (unpublished).

${ }^{52}$ G. Rudnick and G. Gaspari, J. Phys. A 19, L191 (1986).

${ }^{53}$ D. Boris and M. Rubinstein, Macromolecules 29, 7251 (1996).

${ }^{54}$ T. J. Prosa, B. J. Bauer, E. J. Amis, D. A. Tomalia, and R. Scherrenberg, J. Polym. Sci., Part B: Polym. Phys. 35, 2913 (1997).

${ }^{55}$ A. Topp, B. J. Bauer, J. W. Klimash, R. Spindler, D. A. Tomalia, and E. J. Amis, Macromolecules 32, 7226 (1999).

${ }^{56}$ See EPAPS Document No. E-JCPSA6-130-059913 for three dimensional $x y z$ structure of the meso model. For more information on EPAPS, see http://www.aip.org/pubservs/epaps/html. 